\title{
Electrophoretic Karyotype of the Pathogenic Yeast Cryptococcus neoformans
}

\author{
By ITZHACK POLACHECK* AND GAIL A. LEBENS \\ Department of Clinical Microbiology, The Hebrew University-Hadassah Medical School, \\ Hadassah Medical Center, PO Box 12000, Jerusalem 91120, Israel
}

(Received 14 June 1988; revised 19 August 1988; accepted 27 September 1988)

The electrokaryotype of the pathogenic yeast Cryptococcus neoformans is described for the first time. Three different patterns were seen: $(a)$ serotypes B and C (variety gattii) are similar and consist of nine chromosome mobility groups of $>580 \mathrm{~kb} ;(b)$ serotype A (variety neoformans) revealed eight chromosome-like groups $>700 \mathrm{~kb} ;(c)$ serotype $\mathrm{D}$ (the second serotype of variety neoformans) not only differs from those described above, but each $\mathrm{D}$ isolate tested showed a different distribution of bands. The discrepancy, and the importance of electrokaryotyping as a taxonomic tool, are discussed.

\section{INTRODUCTION}

Cryptococcus neoformans is a pathogenic yeast that causes cryptococcosis, growing preferentially in the central nervous system. This disease has a high mortality rate (Butler et al., 1964 ) and is now occurring with increasing frequency among AIDS patients. According to a recent survey in the USA, $8.6 \%$ of AIDS victims developed cryptococcosis (Zuger et al., 1986) and in African AIDS cases it is one of the most commonly observed opportunistic infections (Quinn et al., 1986).

$C$. neoformans is an encapsulated basidiomycete with a known sexual stage and life cycle (Kwon-Chung, 1976a, $b$ ). There are two described taxonomic varieties of $C$. neoformans: var. neoformans, designated as either serotype A or D; and var. gattii, designated as serotype B or C (Kwon-Chung et al., 1982a). Although extensive virulence studies on this yeast have been reported (Kwon-Chung et al., 1982b; Kwon-Chung \& Rhodes, 1986; Polacheck et al., 1982; Polacheck \& Kwon-Chung, 1988; Rhodes et al., 1982), our knowledge of its genetic organization is currently limited to mutant isolation (White \& Jacobson, 1985) and some genetic complementation tests (Whelan \& Kwon-Chung, 1986).

By contrast, the genetics of the ascomycete Saccharomyces cerevisiae has been extensively studied. Chromosome mapping and genetic analysis indicate that the genome consists of 17 linkage groups. That 16 of these groups actually represent distinct chromosomes was confirmed by orthogonal field alternation gel electrophoresis (OFAGE) (Carle \& Olson, 1985).

We have modified and adapted the OFAGE technique described for Schizosaccharomyces pombe (Smith et al., 1987) to separate the chromosomal mobility groups of the four serotypes of C. neoformans. Determining the number of chromosomes in this important pathogen would have taxonomic value and would also permit the mapping of chromosome-specific probes for genetic analysis.

\section{METHODS}

Strains. All the strains used in this work were kindly provided by Dr I. F. Salkin (Wadsworth Center for Laboratory Research, New York State Department of Health, Albany, New York, USA) and by Dr K. J. KwonChung (LCI, NIAID, NIH, Bethesda, Maryland, USA) (Table 1). The isolates were taken from diverse sources,

Abbreviation: OFAGE, Orthogonal field alternation gel electrophoresis. 
Table 1. Yeast strains used in this study

Strain Serotype Source*

Saccharomyces cerevisiae

2012

Cryptococcus neoformans

var. gattii

191

CP110

298

444

997

365

ATCC no. +

Cryptococcus neoformans

var. neoformans

372

38-1

162

160

$174 \mathrm{~A}$

12

430

B-3501

433

161

157

$\begin{array}{ll}\text { C } & \text { CSF; NIH } \\ \text { C } & \text { CSF; Oklahoma } \\ \text { C } & \text { CSF; California } \\ \text { B } & \text { Sputum; NIH } \\ \text { B } & \text { CSF; Brazil } \\ \text { B } & \text { CSF; Thailand }\end{array}$

32608

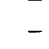

32609

$-$

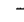

Pigeon droppings; NIH $\quad 34870$

CSF; NIH 34869

CSF; NYS

Blood; NYS

CSF; NIH

34869

Human bone lesion; NIH 28957

Pigeon droppings; Denmark 28958

SB from cross $12 \times 430$

Pigeon nest; NIH 34875

Sputum; NYS

CSF; NYS

\footnotetext{
* CSF, cerebrospinal fluid. SB, single basidiospore; NIH, National Institutes of Health; NYS, New York State Department of Health.

$\dagger$ ATCC, American Type Culture Collection.
}

and were identified using conventional procedures (Kwon-Chung \& Fell, 1984). They all grew at $37^{\circ} \mathrm{C}$, were ureaand inositol-positive and nitrate-negative, and produced a brown pigment when grown on Niger seed agar. Furthermore, all were identified with the API $20 \mathrm{C}$ test and showed similarity in the assimilation pattern of at least 19 carbohydrates. Some of them showed variation, in assimilation of $L$-arabinose. The isolates were serotyped both with specific antibody reagents and with canavanine-glycine-bromthymol blue agar. All isolates were maintained on Sabouraud dextrose agar until used.

DNA preparation. Chromosomal DNAs were prepared from Saccharomyces cerevisiae by the method of Smith $e t$ al. (1987) and from $C$. neoformans as follows. An inoculum from an overnight culture was transferred into $100 \mathrm{ml}$

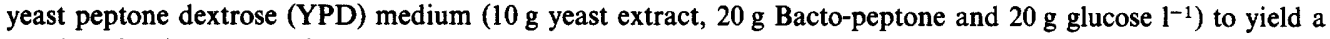
density of $10^{4}$ yeasts $\mathrm{ml}^{-1}$.

The yeast cells were grown overnight at $30^{\circ} \mathrm{C}$ on a shaker up to $2 \times 10^{7}$ yeasts $\mathrm{ml}^{-1}$ (early exponential phase). Approximately $10 \mathrm{ml}$ of cells was then washed twice in $50 \mathrm{mM}$-EDTA pH 7.5 and resuspended in $1 \mathrm{ml} 20 \mathrm{~mm}$ -

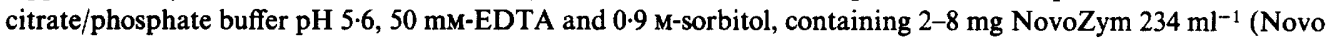
Industrias, Bagsvaerd, Denmark). This suspension was incubated in a water bath at $30^{\circ} \mathrm{C}$. A $10 \mu \mathrm{l}$ sample was removed every $15 \mathrm{~min}$ and mixed with an equal volume of $0.25 \mathrm{M}$-EDTA, $1 \%(\mathrm{w} / \mathrm{v}) \mathrm{SDS}$ and checked for lysis under a phase-contrast microscope. When total lysis had occurred it was assumed that there was $90-100 \%$ protoplast formation; this usually occurred within $45-60 \mathrm{~min}$. Alternatively, the protoplasts were stained with $0.1 \%$ methylene blue in $50 \mathrm{~mm}$-phosphate buffer $\mathrm{pH} 7.0$.

A $1 \mathrm{ml}$ volume of protoplasts was mixed with $1 \mathrm{ml} 1 \%$ low-melting-point agarose (BRL) made up in $0.125 \mathrm{M}$ EDTA pH 7.5. Then $1 \mathrm{ml}$ of this mixture was placed in each of two wells of a 24-well tissue culture cluster (well diameter $16 \mathrm{~mm}$ ) and allowed to set. Each block was cut into smaller pieces and these were mixed in a sterile tube with $5 \mathrm{ml} 0.25 \mathrm{M}$-EDTA pH 7.5, $1 \%$ (w/v) SDS and incubated at $60^{\circ} \mathrm{C}$ for $2 \mathrm{~h}$. After incubation, the gel pieces were transferred to $3-5 \mathrm{ml} \mathrm{0.5} \mathrm{M-EDTA} \mathrm{pH} \mathrm{9,1 \% (w/v)} \mathrm{sarkosyl} \mathrm{and} 1 \mathrm{mg}$ proteinase $\mathrm{K} \mathrm{ml}^{-1}$ (Sigma) and incubated for $24 \mathrm{~h}$ at $50^{\circ} \mathrm{C}$. This step was repeated with a fresh solution and samples were stored at $4{ }^{\circ} \mathrm{C}$ in $0.5 \mathrm{M}$ EDTA pH 9. The modified method gave no significant background of partially degraded DNA molecules when used in OFAGE analysis.

OFAGE analysis. All the separations were carried out on $24 \mathrm{~cm} \mathrm{1.5 \% ,100} \mathrm{ml} \mathrm{agarose} \mathrm{slab} \mathrm{gels} \mathrm{(Pharmacia}$ agarose NA) with wells measuring $5 \times 3 \times 7 \mathrm{~mm}$. The apparatus used was constructed in our institution according to the specifications of Carle \& Olson (1984), with some modifications to the cooling system. Gels were 
cast and run in $0.5 \times$ TBE buffer $(10 \times$ TBE is $0.89 \mathrm{M}$-Tris base, $0.89 \mathrm{M}$-boric acid, $25 \mathrm{~mm}$-EDTA, pH 8$)$. This buffer was maintained at $12-15^{\circ} \mathrm{C}$ by circulating cooled water through glass spirals positioned in the tank on either side of the cooled gel platform. In addition the electrophoresis buffer was constantly circulated through the electrophoresis chamber with the aid of an external peristaltic pump.

Gels were run at $300 \mathrm{~V}$ (constant voltage) with a pulse time of $55 \mathrm{~s}$ for a period of $25 \mathrm{~h}$. They were then removed and stained in $0.5 \mathrm{mg}$ ethidium bromide $\mathrm{ml}^{-1}$ for $45 \mathrm{~min}$ and after this destained for up to $2 \mathrm{~d}$ in numerous changes of $0.5 \times$ TBE buffer. Photographs were taken with Polaroid type $667 \mathrm{film}$ using an ultraviolet transilluminator (Camag Reprostar, Switzerland).

\section{RESULTS}

The electrokaryotypes of 17 isolates of $C$. neoformans (Table 1) representing both varieties gattii and neoformans, each with their two respective serotypes, were studied. All the isolates were from diverse sources, having been obtained from different hosts, niches or geographical localities. In order to check the validity of the technique, at least one isolate of each serotype from the American Type Culture Collection was included.

The electrokaryotypes differed among the isolates of $C$. neoformans studied. The main differences were found between the two varieties. Fig. 1 shows the pattern of the chromosome mobility groups of variety gattii (lanes 1 and 2) compared with those of variety neoformans (lanes 3-5).

There was great similarity among the isolates of the variety gattii. The same band pattern was obtained when using isolates from either serotype $\mathbf{C}$ or $\mathbf{B}$ of this variety (Fig. 1, lanes 1 and 2). Each lane has nine bands, the smallest being around $580 \mathrm{~kb}$ and the largest $>1.6 \mathrm{Mb}$ as estimated from the Saccharomyces cerevisiae marker in lane 6 (Carle \& Olson, 1984; Chu et al., 1986; Mortimer \& Schild, 1985). Isolates 191 and 444 were tested a number of times and the

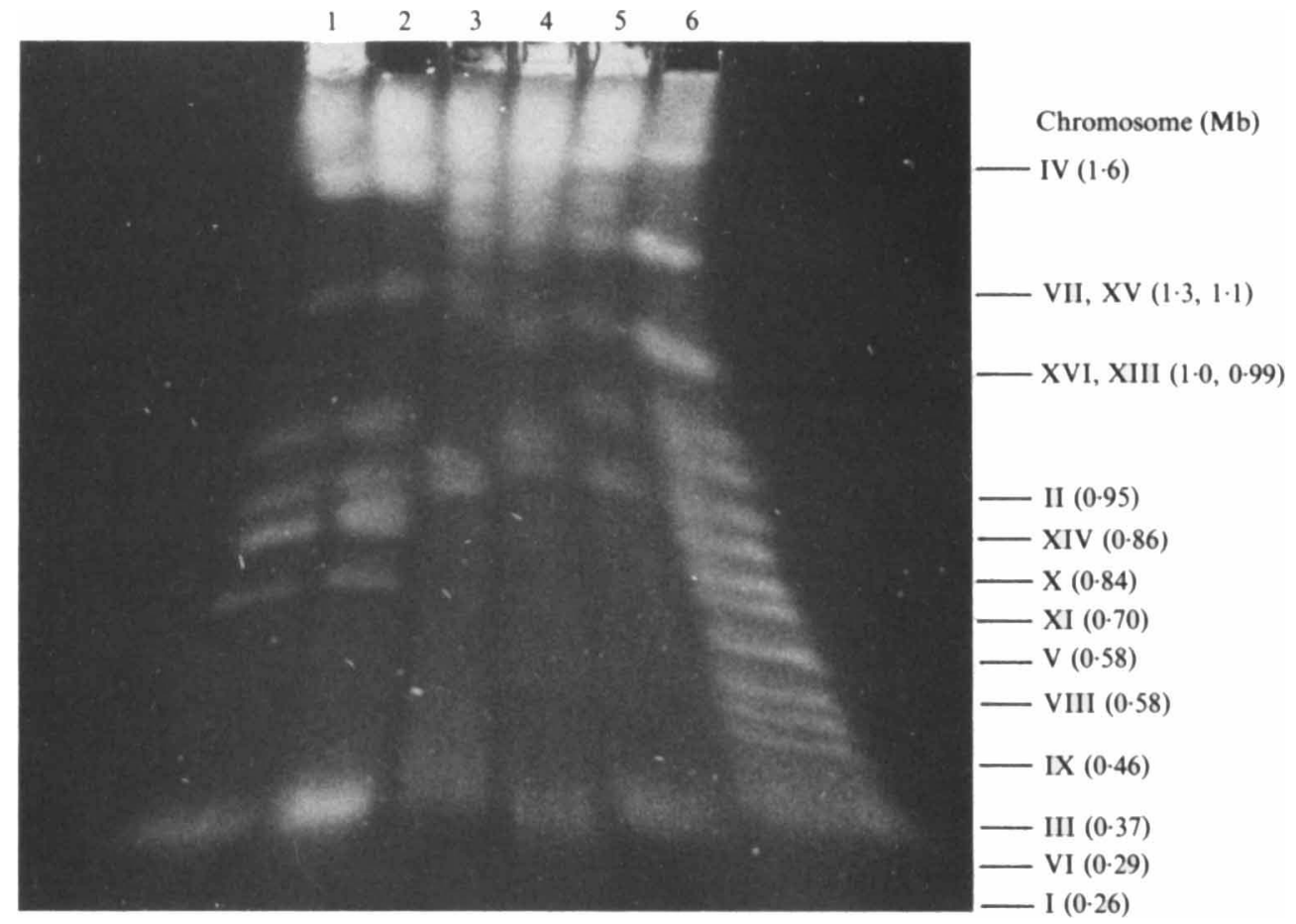

Fig. 1. An OFAGE gel of $C$. neoformans chromosomal DNA. Lanes 1, 2, 3, 4 and 5 represent isolates 191 (serotype C), 444 (serotype B), 372 (serotype A), B-350,1 (serotype D) and 12 (serotype D). Lane 6 represents Saccharomyces cerevisiae 2012, as a marker; the positions and sizes of its 13 chromosome bands are shown on the right. Sizes $\leqslant 0.7 \mathrm{Mb}$ are as determined by OFAGE (Carle \& Olson, 1985); sizes $>0.7 \mathrm{Mb}$ are current physical estimates (Mortimer \& Schild, 1985). 

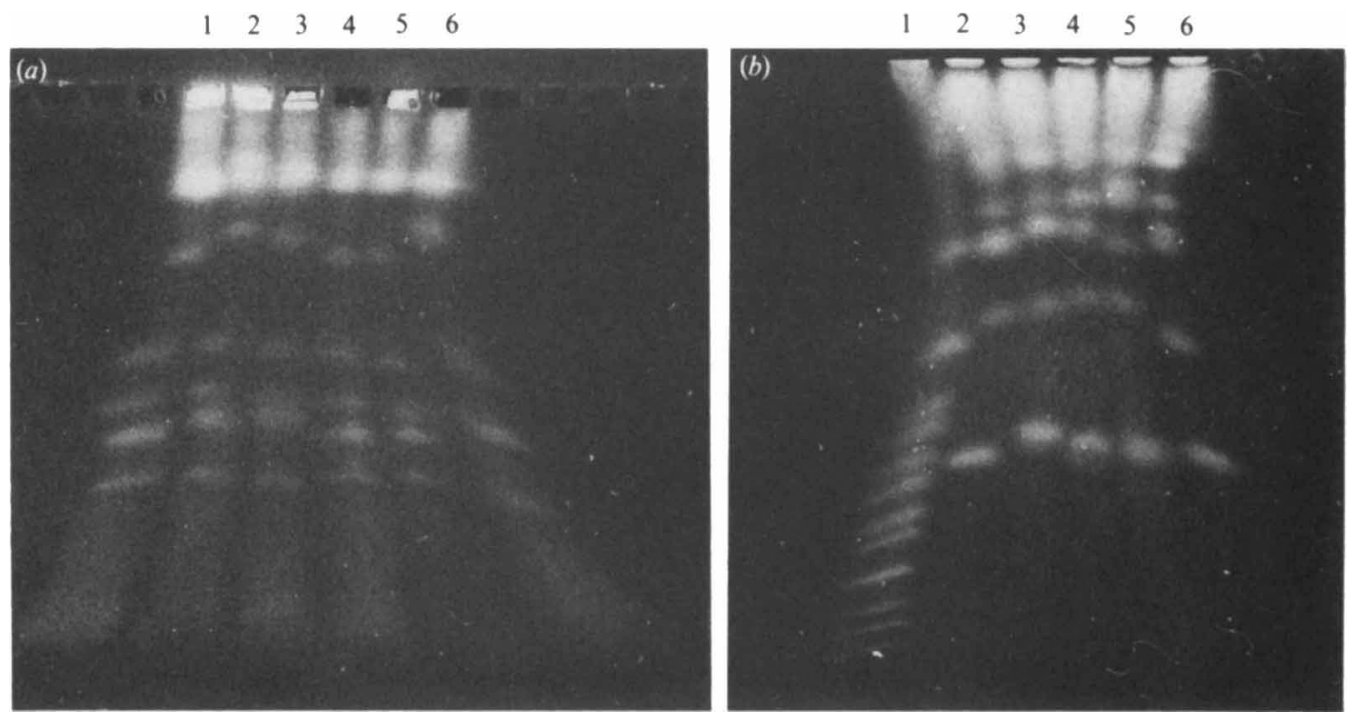

Fig. 2. (a) Electrokaryotype of $C$. neoformans var. gattii. Lanes 1, 2 and 3 represent isolates of serotype C: 191, CP110 and 298, respectively. Lanes 4, 5 and 6 represent isolates of serotype B: 444, 997 and 365, respectively. (b) Electrokaryotype of serotype A of $C$. neoformans var. neoformans. Lanes 2-6 represent isolates 38-1, 160, 372, 174A and 162, respectively. Lane 1 represents Saccharomyces cerevisiae 2012.

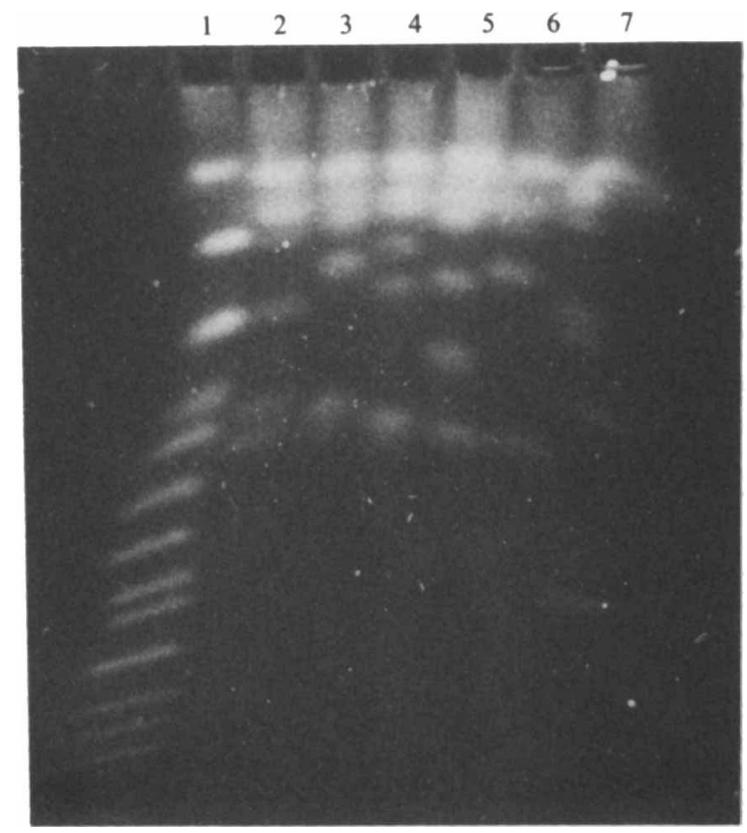

Fig. 3. Electrokaryotype of serotype $\mathrm{D}$ of $C$. neoformans var. neoformans. Lanes 2-7 represent isolates B-3501, 430, 433, 12, 161 and 157, respectively. Lane 1 represents Saccharomyces cerevisiae 2012.

results were consistent with those shown in Fig. 1. In addition we ran gels of a number of different isolates of serotypes B and C (listed in Table 1): all showed similar patterns with respect to the size and distribution of the chromosomal DNA elements (Fig. 1, lanes 1 and 2; Fig. 2a).

The variety neoformans showed a different and less consistent pattern. There was apparent 
similarity among isolates of serotype A (Fig. $2 b$ ), while each of the six isolates of serotype D showed a different electrophoretic karyotype (Fig. 1, lanes 4 and 5; Fig. 3). Comparison of the running of the marker lanes in Figs 2 and 3 shows that the gels are comparable. With serotype A (Fig. 1, lane 3), there appear to be eight bands (the lower band is a doublet, as confirmed on other gels) the smallest being approximately $700 \mathrm{~kb}$ and the largest $>1.6 \mathrm{Mb}$. Lanes 4 and 5 of Fig. 1 show isolates of serotype D. These also appear to have eight bands in a range of 580 to $>1600 \mathrm{~kb}$; however, the distribution of the bands is not the same as that seen in serotype $\mathrm{A}$. To pursue this point further we ran gels of a number of isolates of both serotypes (listed in Table 1); Fig. 2(b) (lanes 2-6) shows the chromosomal distribution of five isolates of serotype A; they all appear quite similar. In contrast, each of six isolates of serotype D (Fig. 3, lanes 2-7) demonstrates a unique banding pattern. We have found no two isolates of serotype $D$ that give the same band distribution. The good separation of the marker bands in Fig. 3 (lane 1) indicates that the differences found in band distribution are not a result of gel distortion.

In addition to the data presented in Figs 1-3, four more isolates of each serotype from diverse sources were tested for the electrophoretic karyotype (data not presented). All of them showed patterns similar to those described above.

\section{DISCUSSION}

C. neoformans is an encapsulated yeast belonging to the class Basidiomycetes, and has a different cell wall from other yeasts such as Saccharomyces cerevisiae, Candida albicans and Schizosaccharomyces pombe. We thus modified the published methods of DNA extraction (Carle \& Olson, 1985; Smith et al., 1987) by introducing a novel protoplast formation step. NovoZym was the only cell-wall-hydrolysing enzyme tested that produced satisfactory results. This method allowed chromosomes of $C$. neoformans to be visualized for the first time. Furthermore, the lysis steps outlined in the procedure of Smith et al. (1987) gave higher yields of DNA with this yeast than did the methods of Carle \& Olson (1985) that we had tried previously.

A diversity of isolates was chosen for this study, rather than a group with similar origins and physiology. However, all the isolates show similarities and represent the range of characteristics within the current concept of the species complex (Kwon-Chung \& Fell, 1984). The serotyping of $C$. neoformans is based mainly on the polysaccharide structure of the capsule (Bhattacharjee $e t$ $a l ., 1984)$. It is believed that there are discrepancies among the serotyping results from different laboratories. However, we found no major differences between the isolates obtained from the mycological laboratory in NIH and those obtained from the reference laboratory in New York State.

Our results demonstrate that $C$. neoformans possesses its own unique distribution of chromosome mobility groups when compared to those of the various species of yeast already published. Schizosaccharomyces pombe is reported to have only three extremely large chromosomes estimated at $3 \mathrm{Mb}, 5 \mathrm{Mb}$ and $7 \mathrm{Mb}$ (Vollrath \& Davis, 1987). The diploid yeast Candida albicans is reported to have a somewhat variable number of bands, between five and nine (Lott et al., 1987; Magee \& Magee, 1987; Snell \& Wilkins, 1986). Saccharomyces cerevisiae chromosomal DNA has been resolved to 12-15 distinct bands of known sizes ranging from $260 \mathrm{~kb}$ (chromosome I) to approximately 2.2 Mb (chromosome XII) (Carle \& Olson, 1984; Chu et al., 1986; Mortimer \& Schild, 1985). The Saccharomyces cerevisiae strain used as a marker in this study gave 13 bands.

Our study of the $C$. neoformans electrokaryotype revealed nine bands in the variety gattii and eight bands in the variety neoformans. These bands were well distributed over the size range of most of the Saccharomyces cerevisiae markers. This is in marked contrast to the band distribution seen in Schizosaccharomyces pombe and the majority of Candida species, where the chromosomes appear to be very large (Magee \& Magee, 1987; Smith et al., 1987). The definitive chromosome numbers for $C$. neoformans are not clear. Our gel system does not resolve chromosome XII of Saccharomyces cerevisiae (2.2-2.5 Mb) (Chu et al., 1986; Vollrath \& Davis, 1987). In addition, all the OFAGE gels appeared to have significant fluorescence at a position above the largest size marker (chromosome IV of Saccharomyces cerevisiae), indicating that large bands of $C$. neoformans may still be concealed at the top of the gel. 
Our results show that isolates of different varieties of $C$. neoformans have different chromosome patterns, a feature unique to this species. In other yeasts such as Candida species the electrophoretic karyotype is species-specific (Lott et al., 1987). The electrophoretic karyotype should perhaps be included as a taxonomic character for yeasts in the same manner as the $\mathrm{G}+\mathrm{C}$ content, since it represents a major biological property. Our results would thus suggest that taxonomists should reconsider the status of the two varieties of $C$. neoformans. Although there is a significant DNA sequence homology between the two varieties (Kwon-Chung \& Fell, 1984), they may represent two different species.

According to the literature, a cross between serotypes $A$ and $D$ always results in $D$ phenotypes among the progeny (Bhattacharjee et al., 1984). This may indicate dominance of the D phenotype; however, in contrast to this phenotypic dominance, serotype $\mathrm{D}$ is rarely found in clinical specimens compared to serotype A, which accounts for $75 \%$ of cryptococcosis in the USA (Kwon-Chung \& Bennett, 1984). In general, when strains of opposite mating types of the different $C$. neoformans varieties are crossed no fertile progeny are found, with one exception - a cross between serotypes $B$ and $D$ gives about $30 \%$ viable progeny, all of which are of the D phenotype (Kwon-Chung et al., 1982a; Kwon-Chung \& Fell, 1984). The above data may explain why each isolate of serotype $\mathrm{D}$ tested gave a different chromosome band pattern. Some of the serotype D strains are perhaps the result of a cross between the two mating types of serotype D, while others may be the progeny of crosses between serotypes A and D or B and D.

The data presented here give an overall view of the physical structure of the cryptococcal genome. The use of isolates from diverse sources, including isolates from an international culture collection, and the similar pattern of the running of the Saccharomyces cerevisiae marker in each gel, indicates the reproducibility and validity of the OFAGE technique. We believe that the electrophoretic karyotype of pathogenic fungi will become an important taxonomic tool.

This work was partially supported by a grant from the Kuvin Center for the study of Infectious and Tropical Diseases. We thank I. F. Salkin and K. J. Kwon-Chung for kindly providing the strains. We especially wish to thank J. Shlomai for his help.

\section{REFERENCES}

Bhattacharjee, A. K., Bennett, J. E. \& GlaudeMANS, C. P. J. (1984). Capsular polysaccharides of Cryptococcus neoformans. Reviews of Infectious Diseases 6, 619-642.

Butler, W. T., Alling, D. W., Anderson, J. \& UTz, J. P. (1964). Diagnostic and prognostic value of clinical and laboratory finding in cryptococcal meningitis. New England Journal of Medicine 270, 59-67.

Carle, G. F. \& Olson, M. V. (1984). Separation of chromosomal DNA molecules from yeast by orthogonal field gel electrophoresis. Nucleic Acids Research 12, 5647-5664.

Carle, G. F. \& Olson, M. V. (1985). An electrophoretic karyotype for yeast. Proceedings of the National Academy of Sciences of the United States of America 82, 3756-3780.

ChU, G., Vollrath, D. \& Davis, R. W. (1986). Separation of large DNA molecules by contourclamped homogeneous electric fields. Science 234, $1582-1585$.

KWON-CHUNG, K. J. (1976a). Morphogenesis of Filobasidiella neoformans, the sexual state of Cryptococcus neoformans. Mycologia 688, 821833.

Kwon-Chung, K. J. (1976b). A new species of Filobasidiella, the sexual stage of Cryptococcus neoformans B and C serotypes. Mycologia 689, 942946.
Kwon-Chung, K. J. \& Bennett, J. E. (1984). Epidemiologic differences between the two varieties of Cryptococcus neoformans. Journal of Epidemiology 120, $123-130$.

Kwon-Chung, K. J. \& Fell, J. W. (1984). Filobasidiella Kwon-Chung. In The Yeasts: a Taxonomic Study, 3rd edn, pp. 472-482. Edited by N. J. W. Kreger-van Rij. Amsterdam: Elsevier Science Publishers.

KWon-Chung, K. J. \& RHodes, J. C. (1986). Encapsulation and melanin formation as indicators of virulence in Cryptococcus neoformans. Infection and Immunity 51, 218-223.

Kwon-Chung, K. J., Bennett, J. E. \& Rhodes, J. C. (1982a). Taxonomic studies on Filobasidiella species and their anamorphs. Antonie Van Leeuwenhoek 48, 25-38.

Kwon-Chung, K. J., Polacheck, I. \& Popkin, T. J. (1982b). Melanin-lacking mutants of Cryptococcus neoformans and their virulence for mice. Journal of Bacteriology 150, 1414-1421.

LotT, T. J., BoIron, P. \& Reiss, E. (1987). An electrophoretic karyotype for Candida albicans reveals large chromosomes in multiples. Molecular and General Genetics 209, 170-174.

Magee, B. B. \& Magee, P. T. (1987). Electrophoretic karyotypes and chromosome numbers in Candida species. Journal of General Microbiology 133, 425430. 
MoRTIMER, R. K. \& SCHILD, D. (1985). Genetic map of Saccharomyces cerevisiae, edition 9. Microbiological Reviews 49, 181-212.

Polacheck, I. \& K Won-Chung, K. J. (1988). Melanogenesis in Cryptococcus neoformans. Journal of General Microbiology 134, 1037-1041.

Polacheck, I., Hearing, V. J. \& KWon-Chung, K. J. (1982). Biochemical studies of phenoloxidase and utilization of catecholamines in Cryptococcus neoformans. Journal of Bacteriology 150, 1212-1220.

Quinn, T. C., Mann, J. W., Curran, J. W. \& Piot, P. (1986). AIDS in Africa: an epidemiologic paradigm. Science 234, 955-963.

Rhodes, J. C., Polacheck, I. \& Kwon-Chung, K. J. (1982). Phenoloxidase activity and virulence in isogenic strains of Cryptococcus neoformans. Infection and Immunity 36, 1175-1184.

Smith, C. L., Matsumoto, T., Niwa, O., KlCo, S., FAN, J. B., YANAGIDA, M. \& CANTOR, C. R. (1987). An electrophoretic karyotype for Schizosaccharo- myces pombe by pulsed field gel electrophoresis. Nucleic Acids Research 15, 4481-4489.

SNELL, R. G. \& WILKINS, R. J. (1986). Separation of chromosomal DNA molecules from Candida albicans by pulsed field gel electrophoresis. Nucleic Acids Research 14, 4401-4406.

VollRath, D. \& Davis, R. W. (1987). Resolution of DNA molecules greater than 5 megabases by contour-clamped homogenous electric fields. Nucleic Acids Research 15, 7865-7876.

WhElan, W. L. \& KWON-ChUNG, K. J. (1986). Genetic complementation in Cryptococcus neoformans. Journal of Bacteriology 166, 924-929.

White, C. W. \& JACOBSON, E. S. (1985). Occurrence of diploid strains of Cryptococcus neoformans. Journal of Bacteriology 161, 1231-1232.

Zuger, A., Louie, E., Holzman, R. S. Simberkoff, M. S. \& RaHAL, J. J. (1986). Cryptococcal disease in patients with the Acquired Immunodeficiency Syndrome. Annals of Internal Medicine 104, 234-240. 